\title{
Conceptualizing Interpersonal Interruption Management: A Theoretical Framework and Research Program
}

\author{
Sukeshini A. Grandhi \\ New Jersey Institutes Of Technology \\ sg49@njit.edu
}

\author{
Quentin Jones \\ New Jersey Institutes Of Technology \\ qgjones@acm.org
}

\begin{abstract}
Previous research exploring interpersonal-technologymediated interruptions has focused on understanding how the knowledge of an individual's local context can be utilized to reduce unwanted intrusions by employing sensor and agent technology to detect and manage their interruptions. However, this approach has produced limited benefit for users because it fails to take into account who the interruption is from or what it is about. To address this deficiency a theoretical framework and associated research program is presented to provide a fresh perspective on design of interruption management tools.
\end{abstract}

\section{Introduction}

Numerous devices and appliances have found their way to our homes, offices and even our bodies. Many workplace domains encourage and embrace technology to benefit from dynamic and up to date information availability and exchange. However, the information these technologies bring when inappropriate, irrelevant, or excessive, creates a classic modern age problem of interruptions affecting individual productivity. This technological predicament highlights the need for understanding the structure of how people manage interruptions so as to harness the benefits while minimizing the costs.

Researchers are keen to find ways to design Information Systems that ameliorate the management of unwanted interruptions. The term "interruption" when used in the realm of individuals' behavior often brings to mind negative connotations associated with unwanted interjections into cognitive and social processes. Since the early 1920 s researchers in psychology have typically explored attention and interruptions in terms of limited attention capacity models [6][23]. Similarly, a number of CSCW researchers extended this work to look at the impact of interruptions on individual and collaborative computer supported tasks [6][27][36]. In a similar vein, and not surprisingly, much of the current interruptibility research effort is directed by those exploring how sensor [10][18] and agent technology [26] can be used to mitigate the negative effects of interruptions by identifying those situations when an interruption will be least cognitively taxing or socially intruding. However, this paradigm of focusing on social and cognitive factors localized to the individual being interrupted, understates the impact of the source (who) and content (what) of interruptions on interruption management decisions.

We know from workplace studies that in some organizations interruptions are a way of life, that are often sought and even desired due to factors such as interdependencies of work activities, cooperation and sociality of work, and organizational reward systems[21][29][31]. In such cases a person might be open to being interrupted by a particular colleague even if he/she is cognitively and socially overloaded. In our own everyday communication practices it is easy to think of situations in which we may not want to deal with an interruption from person A for any reason but be willing to accept an interruption from person $B$ even if our cognitive or social space is disrupted. Therefore, if we are to gain an understanding of real world interruptibility or interruption management, over and above the cognitive and social aspects surrounding the person being interrupted (interruptee) we need to gain a richer and deeper understanding of the impact of what we term as the 'relational context.' This context encompasses all aspects between the interrupter and the interruptee such as the nature of the relationship, what the interruption is about, under what circumstances is interrupter interrupting, and historic interrupter-interruptee interaction patterns that define the nuances of relationships.

This paper is organized as follows: After briefly reviewing previous approaches to defining and predicting human interruptibility, we highlight the inadequacies of these approaches in providing a complete picture of interruptibility. To address this shortcoming we define relational context and argue why it is vital in understanding interruptibility and 
interruption management. We then present a theoretical framework that provides insight into how information on relational context can be leveraged to help reduce interruptee's uncertainty about an interruption and therefore more accurately predict its value to improve interruption decision making. Once this framework is fully explicated, we conclude with a discussion of how a research program based on this framework will inform design of collaborative systems.

\section{Background}

\subsection{Operationalizing Interruptibility}

Interruptibility is an amorphous term with researchers adopting two fundamentally distinct definitions and associated research paradigms. One school of thought derives the level of interruptibility based on how interruptions impact on task performance and mental workload [2][22][31]. Measures of interruptibility adopted by these researchers are based on how much an interruption negatively affects one's cognitive and social state. Studies on interruptions that took this perspective looked at how interruptions affected task performance with respect to various variables such as the mode of interruption [8] [25], methods of coordination of an interruption [27], complexity of interrupted task [13], relevance of interruption to current task (see [27] for a comprehensive review). Findings of these studies were consistent in that interruptions do have an impact on people's cognition and task performance but whether they had a negative effect or positive effect varied with interruption characteristics such as timing [31], frequency, length and similarity to main task [13].

The second school of thought distinguishes between how interruptions affect one's cognitive/social state and how receptive people are to interruptions. Researchers adopting this paradigm addressed interruptibility by explicitly looking at individuals' self reports on their perceptions of burden/cost [20][21], perceptions of value [24], willingness to be interrupted [18] and responsiveness to an interruption [2]. This definition of interruptibility in interpersonal communication is more fitting since how much it affects one's cognitive/social state, and how willing people are to allow it to affect their cognitive/social state, are two different things. Interruptibility then becomes a conscious choice that people make about their willingness to be interrupted based on who the interruption is and what they think it is about. We will use this definition of interruptibility in the rest of the paper.

\subsection{Predicting Interruptibility}

The key goal of HCI interruptibility research over the past decade has been to understand how to predict interruptibility in order to deploy systems that reduce the likelihood of unwanted interruptions. This work has been further driven by the growth in sensor technology and techniques of data capture/analysis which enable rich modeling of everyday activities. The rationale being that the timing of interruption can be optimized by inferring interruptibility from factors local to the interruptee such as the current activity and the level of involvement in it, location, and presence of people around.

In the desktop environment, considerable effort went in capturing the task of an individual and level of cognitive involvement in tasks. Iqbal et. al. [22] used eye tracking sensor technology to capture pupil dilation as an indicator of mental workload in the desktop environment. They found that a user's mental workload changes among subtasks and decreases at subtask boundaries and hence hypothesize that interruptions should occur at these instances. Hortvitz et. al. [19] captured click streams of high level desktop activities such as timing and pattern of switching between applications and low level activities such as clicking, moving and typing. They were able to predict individuals' perceived interruptibility at $70-87 \%$ accuracy. However these predictive models cannot be generalized as they do not take into account how their responsiveness may change with interruption source or interruption content.

Beyond the task level effects, researchers who embraced aspects of the social surroundings considered inappropriate situations defined by social and organizational norms of appropriate behavior/actions. Once again, researchers used sensors to detect social activity, place of activity, and people around an individual. Fogarty et. al. [10] used sensors to acquire information on social surroundings such as user's position (standing or sitting), voice, presence of guests, and if the door was open or closed. While combinations of these factors have been shown to predict interruptibility at an accuracy of $79.2 \%$, they overlook "who" the interruption is from, reducing the generalizability of their findings. In the mobile environment, Ho and Intille [18] used sensors to detect physical activity transitions as an indicator of receptiveness for interruptions. Kern et. al. [24] also used sensors to distinguish between personal interruptibility factors of the interruptee and social interruptibility which is the impact an interruption has on people in the surrounding environment based on social norms. The knowledge gained from these studies advanced interruption research to the level where 
researchers aimed at predicting opportune moments to interrupt based not only on the individuals cognitive state but also based on the social situation. However like the previous work discussed they also overlook the relational aspects of who the interruption is from, what it is about and how that can change responsiveness.

Expanding the factors that need to be taken into account when predicting interruptibility still further, Horvitz et. al. [20] evaluated real time willingness to be interrupted based on calendar information of meeting attendance and pre-defined user interruptibility levels. They allowed users to define callers' interruption rights based on organizational relationships, activities, and ad hoc groups such as "critical associates" and "close friends". While this work breaks new ground by incorporating relational aspects that has so far been ignored by previous models of interruptibility, it does not adequately explore this space for the following reasons. Firstly, the scope of the study is limited to interruptibility in a narrow social setting of being in a meeting for two individuals. Secondly, the relational aspect used is based on heuristics derived from broadly defined social and organizational groups rather than the nuances of interpersonal relationships.

\subsection{Interruption Context}

The above review of interruptibility research shows how previous efforts have focused on aspects local to the interruptee, either on what a person is doing at the time of an interruption and/or the interruptee's social surroundings including who a person is co-located with. This perspective of sensing and reacting to aspects local to the interruptee draws upon the work in context-aware computing research. Context-aware applications aim to support users by semiautomatically catering to their needs through sensing and reacting to their environments. In this case the sensing is of activities associated with different levels of interruptibility and the user support is in terms of interruption management.

There are however two fundamental problems with the use of the current 'context-aware computing' paradigm to interruptibility. The first is in terms of the understanding of 'context'. In the first work that used the term context-aware, Schilit and Theimer [32] defined context as location, identities of nearby people and objects, and the changes to those objects. Shortly thereafter this definition was narrowed to include "where you are [or the application], who you are with, and what resources are nearby" [33]. Dey [10] expanded the definition to "any information that can be used to characterize the situation of an entity. An entity is a person, place, or object that is considered relevant to the interaction between a user and an application, including the user and the applications themselves". In applying the notion of context-awareness to interruptibility, researchers have considered the primary entity under investigation to be the interruptee, what he/she is doing, who he/she is with, who he/she is collocated with [12][19][22][24]. It is assumed that, when the interruptee entity is properly characterized, interruptibility can be effectively modeled and effective interruption tools can be provided.

This perspective ignores the reality of the everyday interruptions that result from interpersonal communication from various individuals, where clearly "receptiveness" relates not only to interruptee's mental state, activities and social surrounding, but also on several factors not local to the interruptee such as who the interruption is from, the relationship between the interrupter and interruptee, what the interruption is about, activity and location of the interrupter, and the level of importance/urgency to both the interrupter and interruptee. In other words, the interruption context should not be viewed solely in terms of aspects used to characterize the environment of previously developed context-aware applications such as location, identities of nearby people and objects, and changes to those objects, but all aspects between the interrupter and the interruptee including what the interruption is about.

The second weakness in applying the current context-aware paradigm to interruptibility is the emphasis on the design of applications that semiautomatically manage responses based on the relevant environmental context. In this case, this means determining semi-automatically when an interruption should be allowed for the user. As a result the majority of interruptibility research aims at designing contextaware applications that can determine the appropriate instances to prevent unwanted interruptions. Such a position is prejudicial and gets researchers to think about how to take control away from the individual and assumes that the rich context can be understood by software agents. In reality, these agents utilize decision rules that react to data gathered and aggregated on environmental factors surrounding an individual. Unfortunately, the appropriateness of the situation is still determined by the individual as it is based on one's perception. For example, a context aware cell phone may detect loud music and voices surrounding an individual but it may not determine if a phone call from an overseas relative should be allowed or not. Thus even though who the interrupter is and what the interruption is about do not characterize the context of the interrupter entity as defined by the context-aware systems, they still influence the interuptee's responsiveness to the interruption. Hence they become relevant to the context of the individual's response 
decision. This highlights how interruption "context" is different from that typically being dealt with by context-aware systems. Thus rich notions of "context" and "awareness" are concepts that are very differently understood by people from how they are being instantiated in the context-aware systems [11]. This calls for system design that recognizes and supports the use of relational aspects of the give and take between interrupter and interruptee.

To some extent the shortcomings resulting from applying the current context-aware computing paradigm to interruptibility can be addressed by 1) more richly categorizing the interruption context and 2) moving the emphasis from modeling interruptibility for agent control to bringing control back to human decision making. In this effort we subdivide the interruption context to three components:

- Cognitive context which is all aspects that encompass the interruptee's cognitive level of involvement in tasks and how it affects task performance [27][31][36];

- Social context which is all aspects that encompass the interruptee's physical environment surrounding of an individual as understood in a social sense such the place the interrupter is in, other people present and their relationship with the interruptee, and the nature of social activity occurring there [10][18][26]; and

- Relational context which is all aspects between the interrupter and the interruptee such as the nature of the relationship, what the interruption is about, under what circumstances is interrupter interrupting, and historic interrupter-interruptee interaction patterns that define the nuances of relationships.

Current models that predict one's interruptibility using social context or cognitive context paradigms are limited in providing a complete picture of people's willingness to respond to an interruption The handful of prediction models that have incorporated aspects of relational context have limited this to broad affiliations of the interrupter such as friendships, workrelationships and kinship ties [9][19]. Below we illustrate how assessing one's willingness to respond to an interruption based on these models can be misleading, using a common interpersonal communication scenario.

Sally is a working mother, deeply involved in a task with a deadline three hours away. Her cell phone rings and she ignores the call. After twenty minutes her cell phone rings again, and she checks the caller ID. Noting it's from her colleague Kara with who she made plans this morning to meet for drinks after work, Sally ignores it allowing it to go to the voicemail. At this time she also checks the previously ignored call and after noting it was from her husband, she decides to return the call after the deadline. After five minutes, Sally's phone rings again and she checks the caller ID and notes it is from her daughter's daycare. Concerned that her daughter is sick, she answers the call immediately. She is relieved when she realizes that the daycare was calling to remind her about a late payment. Three hours later she checks her voicemail to hear her friend Kara's message. In her voice message Kara requests for a quick clarification regarding the budget for another project. Wishing she knew what Kara's reason was at the time of call so that she could have avoided assuming the call was about after work drinks plan, Sally calls Kara right away.

From the above scenario one can deduce that calls to Sally have many characteristics that influence her decision to respond immediately or defer response for a later time. Examining the above scenario from the cognitive context interruptibility paradigm, one would predict that Sally is not interruptible by any incoming phone call due to her high level of cognitive involvement in her work. However, the scenario highlights a fundamental weakness in this approach by emphasizing how Sally's disposition towards interruptibility changes as soon as she gets a call from the daycare. This call is unusual, unexpected, and occurs at a time when her daughter is in the day care, all of which increase her uncertainty and anxiety associated with the call. Sally's uncertainty about the call increases further because of the call she missed from her husband that she has no information about. As a result, she makes a wrong deduction about the importance and urgency of the call from the daycare. that influences her decision to answer it. In contrast, a call from Kara in light of the impending plans they had for the evening led Sally to ignore the call. Had she known the real reason she would have picked up the call from Kara. This scenario illustrates how Sally's decisions vary with the caller identities and perceived reason for each call even as her mental workload remains roughly constant during the period of the three incoming calls. In other words, task based mental workload information on its own cannot reliably detect Sally's interruptibility disposition in regards to these phone calls.

Examining the scenario from the social context interruptibility paradigm also fails to provide a satisfactory account of Sally's receptiveness to interruptions. In this case, Sally is in a work situation, working on a deadline, in her office, and in a work culture that discourages personal calls. Based on these factors one would predict from the 'social context' paradigm that Sally's interruptibility will remain low as long as she is working on the deadline. But clearly, Sally's disposition to certain interruptions changes 
depending on who calls and how often she encounters calls from certain individuals.

Examining the scenario through the lens of social affiliation models also fails to provide an accurate account of Sally's receptiveness to interruptions. Basing it on affiliations such as friendship or workrelationship, one could predict that Sally is interruptible by her colleague who is a critical work associate. Sally however started off being uninterruptible to her colleague due to her previous non-work related interaction history but would have changed her disposition had she known the reason for the call. This scenario highlights how preset and singular categories based on basic affiliations fail to capture the nuances of interpersonal relationships which influence one's interruption handling decisions.

All the above models have been incorporated into interruption management tools, which are utilized to predict an individual's availability and/or willingness to be interrupted [10][18][19][20][24]. However, the above scenario illustrates such naive computation of interruptibility may be inaccurate due to the dynamic nature of relational context which is crucial for interruption management decisions. Some researchers have shown that the relationship between the interrupter and interruptee influences one's interruptibility or response to interruptions. Two separate studies of administrative assistants as mediators of interruptions show that importance of interruption was derived based on relationship between interrupters and interruptees [8][35]. However these studies are limited to a set of very specialized workers who mediate interruptions. This argues the necessity for tools that incorporate a more holistic understanding of how interruption management decisions are made by utilizing not only the social and cognitive context but also a rich account of the relational context.

\section{Interruptibility Framework}

In previous sections we highlighted the gaps in the traditional understanding of interruptibility and hence interruption management resulting from overemphasizing the localized cognitive and social context while largely ignoring the relational context. We now present a theoretical framework that can inform design of interruption management tools in mediated communication.

Interpersonal actions and communication motives are influenced by individual and cultural differences and have been theorized using several ways including symbolic interactions and dramaturgy [6][14]. However, if we assume that human beings are rational decision makers, their willingness to be interrupted will be based on an evaluation of the costs and benefits of responding to an interruption. Using this perspective, this theoretical framework is derived from two rational choice communication theories that aim to explain information seeking behavior in initial social interactions between strangers: 1) Uncertainty Reduction theory (URT), which states that high levels of uncertainty between strangers' prompts them to ask questions of each other thereby reducing uncertainty [5], and 2) Predicted Outcome Value theory (POVT), that posits that the motivation to interact is governed by a desire to maximize relational outcomes [35]. POVT further posits that the outcome value may be positive or negative leading to communication alternatives such as termination, restriction, continuation, expansion, or escalation of the early interaction and relationship. While these theories were originally proposed to provide insight on how people seek information to develop, maintain or terminate a relationship, when used in the realm of interruptibility, together they provide insight on how individuals interpret and respond to an interruption.

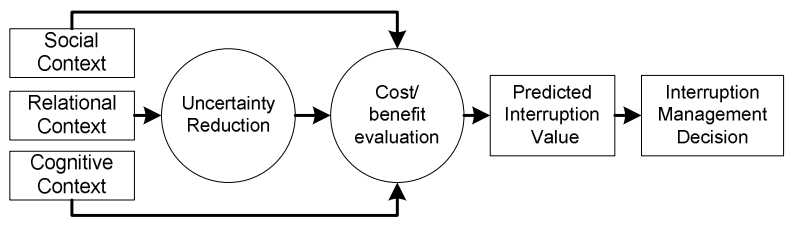

\section{Figure 1: Interruption Management Decision Framework}

When individuals are interrupted by a technologymediated request for interpersonal communication, as rational actors, they attempt to predict the interruption's value. The Predicted Interruption Value (PIV) is the result of a cost/benefit evaluation for the best course of action in response to the interruption, based on cognitive, social, and relational context. While individuals are aware of their own cognitive and social context, their knowledge of the relational context is to some extent unknown. As a result, they will typically try to reduce uncertainty regarding the relational context to derive the PIV. This logic is presented in Figure 1, the interruption management decision framework. The framework recognizes that individuals engage in uncertainty reduction as a means to predict the value of an interruption and that this in turn influences their decision to be interrupted. It also suggests how the presentation of rich relational context information, by reducing uncertainty, can aid in an individual's interruption management decisions. 
From prior work in computer-mediated communication (CMC) and Human Computer Interaction (HCI) we identify three categories of relational context factors of particular importance: 1) Interrupter related information which comprises factors such as who the interruption is from, his or her activity, location, mood, and the nature of relationship with people in the location [10]; 2) Interruption content which comprises factors such as what the interruption is about (analogous to subject fields in emails), how long it may last, and its urgency/importance [9][17][27]; 3) Interrupter-Interruptee interaction history which comprises factors such as how often, for how long, what times the interrupter interrupts, and how many attempts were previously made.

\subsection{Uncertainty Reduction}

The theoretical framework presented in this paper makes it possible to tease out ways in which information on relational context can be used to reduce uncertainty around an interruption thereby enabling an interruptee to more accurately predict the outcome value of an interruption. The relational factors identified above vary with each interruption and the knowledge of these factors influences the interruptee's response to an interruption. However in current CMC systems the relational context knowledge is limited or non existent leading to uncertainty around an incoming interruption as discussed below.

Interrupter Related Information: Uncertainty around interrupter's context in CMC comes from factors such as who the interrupter is and his or her social and cognitive context. Currently identity of the interrupter is limited to information on interrupter identification (ID) in terms of numbers, names, usernames and email addresses. While relationships and affinities to the interrupter are not explicitly provided, they are implicitly derived in the interruptee's mind based on the interrupter's ID information. The implicit derivation however is based on the premise that the user ID presented is that of the person(s) having ownership or user rights to the communication device. The case of multiple users increases the level of uncertainty on who the interrupter is. As far as computer-mediated interrupter's social or cognitive context factors are concerned, currently the interrupter's context while initiating an interruption in terms of the location/place, people in place and social/cognitive activity, mood is not captured to be presented to the interruptees. Some level of uncertainty is reduced if the interruption is via a non-mobile communication device as one can assume that the person is calling from where the device is located. Micro details such as who they are with, their mobility, current task, and scheduled activities can help in reducing uncertainty further. This is a diverse and unexplored area that has many research opportunities to further improve interruption management.

Interruption Content: Uncertainty from on interruption content can arise from lack of knowledge on factors such as importance/urgency of the interruption and length of interruption. Often importance/urgency as perceived by the interrupter is conveyed when engaged in the interruption itself and is rarely communicated ahead of the interruption. Typical asynchronous communication systems such as emails and voicemails allow for the urgency level to be conveyed. Anecdotal experiences shows that individuals can infer urgency/importance based on communication history patterns of the sender such as frequency of and time of day interactions on certain communication media. For some interruptions may be considered important/urgent based on regular communication patterns, for example, a mother who receives a call every evening at $9 \mathrm{pm}$ from her son who is overseas. Alternatively a break in routine communication patterns may be inferred as important, such as an early morning call from a person who rarely calls at that time maybe be perceived as important/urgent. These scenarios highlight that importance/urgency of an interruption currently is implicitly derived or understood or misunderstood. As far as knowing what an interruption is about, it is difficult to know for sure until it is engaged in. However some communication systems like emails allow for a subject line/tag that is synonymous to a headline of a news story. This allows for reduced uncertainty for the receiver by enabling him/her to gauge the value of engaging in the interruption.

Interrupter-Interruptee Interaction History: Interaction patterns such as emails, text messages or phone logs that include times at which they are received and how long they last etc., can be obtained based on the design capability of the device in question. Currently, aggregated/meta information on interaction patterns such as frequency of interruptions, average length, usual times of interruptions are not available explicitly unless actively sought or implied by the user.

The knowledge of the three kinds of relational factors discussed above can help reduce the uncertainty around an interruption leading to a more accurate prediction of the interruption value. The issues of uncertainty raised here are particularly relevant to current cell phone communication where the relational context information provided for incoming calls is limited to the identity of the caller (in other synchronous communications technology such as land phones one could deduce place in which the caller is 
and/or her related activity the place). This could be addressed by leveraging social network and/or location data to provide richer information to the interruptee, or by providing mechanisms for the capture and presentation of information such as call reason, importance and urgency to the interrupter.

Previous support for interruption management has often come in the form of awareness display systems that display the interruptee's current social, cognitive context to the interrupter [30]. This strategy reduces uncertainty to the interrupter but not to the interruptee. Apart from privacy concerns, this design approach raises two fundamental issues: 1) the interruptee's willingness to be interrupted may be independent of their social and cognitive context and may be moderated by who the interruption is from or what it's about; and 2) it leaves the onus of interrupting on the interrupter which may or may not respect or match with the interruptee's desires and inclinations. Measuring the effectiveness of providing the interrupter with information in terms of the degree of agreement between the receiver's desires and callers' decisions, Avarahmi et. al. [1] found that different contextual information generate different perceptions of interruptibility. This lead to a mismatch in judgment of appropriateness for an interruption which in turn led to both under-calling and over-calling in equal severity. In contrast to the awareness display design approach, our theoretical framework has implications for designs that empower the interruptee with the knowledge of the relational context to reduce the uncertainty around an interruption.

Very limited prior work has been done to explore how providing the interruptee with knowledge about the incoming interruption can reduce uncertainty and better predict the value of an interruption. In a study where the sender's identity was revealed in the form of team identity it was found that response improved towards interrupters of the same team [9]. These findings are based on laboratory experiments under conditions of specific help-seeking interruptions similar to that in organizational team communication. Well defined interruptions such as help-seeking tasks however, remove uncertainty pertaining to the reasons and content of the interruption. While this study presents results that have design implications for awareness displays and notifications, it is limited to the focused tasks in controlled settings. Additionally the study's author concedes that while the experimental task incorporated many elements of real organizational work, it does not compare in the level of detail, diversity, and/or complexity to that in real work environments.

\subsection{Predicted Interruption Value}

While prior research has not explicitly defined how interruption value is calculated it has been addressed in different ways. Gruen [17] notes that urgency conveyed about the communication is important in the evaluation and negotiation of interruption in work activities. In a study of email use the importance of message content was measured in terms of perceived importance, time period for which the message will be stored, time proximity of deadlines, and amount of time spent on project/task associated with the message. The findings of this study showed that people considered a message to be important if work related action was required of them [9].

The value of an interruption has also been operationalized by a number of researchers in terms of its cost or burden [10][18][20]. Ho and Intille [18] summarize value in terms of the burden with respect to various factors: activity of the user; utility of a message; emotional state of the user; modality and frequency of the interruption; task efficiency rate; user's perceived authority on interruption, user's previous and future activity; user's social expectation; and history and likelihood of response. Other researchers have built statistical models to infer value based on sensed data from the user's environment such as people, conversation, and applications in use, where a person's self reported interruptibility levels are used as a proxy for costs of interruption at various data points [10][19].

Similar to previous cost benefit models, we define the value of an interruption as a net result of perceived costs and perceived benefits of an interruption. However, our theoretical model shows that the costs and benefits are perceived based not simply on the social and cognitive factors as predominantly considered in previous paradigms but also on relational factors such as interrupter's context, interruption content and history of interrupter-interruptee interaction patterns. Thus we define the predicted value of an interruption (PIV) at time $t$ as

$$
\begin{aligned}
& \operatorname{PIV}(\mathrm{t})=\sum_{\mathrm{i}=1}^{\mathrm{l}}\left(\mathrm{C}_{\mathrm{i}}(\mathrm{t})-\mathrm{B}_{\mathrm{i}}(\mathrm{t})\right) \mathrm{w}_{\mathrm{i}}(\mathrm{t})+\sum_{\mathrm{i}=1}^{\mathrm{m}}\left(\mathrm{C}_{\mathrm{i}}^{\prime}(\mathrm{t})-\mathrm{B}_{\mathrm{i}}^{\prime}(\mathrm{t})\right) \mathrm{w}_{\mathrm{i}}^{\prime}(\mathrm{t})+ \\
& \sum_{\mathrm{i}=1}^{\mathrm{n}}\left(\mathrm{C}_{\mathrm{i}}^{\prime \prime}(\mathrm{t})-\mathrm{B}_{\mathrm{i}}^{\prime \prime}(\mathrm{t})\right) \mathrm{w}_{\mathrm{i}}^{\prime \prime}(\mathrm{t})
\end{aligned}
$$

where $\mathrm{Ci}(\mathrm{t}), \mathrm{Bi}(\mathrm{t})$, and wi(t) are perceived cost, benefit and weight of the ith cognitive factor respectively; $\mathrm{C}_{\mathrm{i}}^{\prime}(\mathrm{t}), \mathrm{B}_{1}^{\prime}(\mathrm{t})$, and $\mathrm{w}_{\mathrm{i}}^{\prime}(\mathrm{t})$ are perceived cost, benefit and weight of the ith social factor; and $\mathrm{C}_{\mathrm{i}}^{\prime}(\mathrm{t}), \mathrm{B}_{\mathrm{i}}^{\prime \prime}(\mathrm{t})$, and $\mathrm{W}_{\mathrm{i}}^{\prime \prime}(\mathrm{t})$ are perceived cost, benefit and weight of the ith relational factor. 
A negative PIV indicates that the perceived costs outweigh the perceived benefits, (i.e. the individual is less likely to respond to the interruption) whereas positive PIV indicates that the perceived benefits outweigh the perceived costs (i.e. the individual is more likely to respond to the interruption). It has to be noted however that PIV is defined as user's perception and the way user assigns weights to each factor is influenced by how s/he perceives the value of these factors. For example, an interruption may affect the productivity of a current task but if the interruptee perceives the importance/urgency to the interrupter to be of higher weight, then the net PIV of responding to the interruption may be positive. Further, the PIV may or may not be accurate as it is evaluated based on the information available to the interruptee at the time of interruption. For example, an individual receiving a call from a colleague may perceive it to have high work related value and low social value. However, it may be that the colleague may have called to ask if the individual would go to a movie with him. The accuracy of PIV is related to the accuracy of interruption information one has at the time of interruption. Our framework shows that we can maximize the accuracy of PIV by providing relevant information and reducing uncertainty around the unknown relational factors of an interruption. Since hindsight is 20/20, the actual/accurate value of an interruption is known only after responding to an interruption. However, the closer the PIV is to the actual interruption value, we hypothesize that the greater the individuals' satisfaction with the decision will be.

\section{Summary and Research Agenda}

The discussion of interruptibility research outlined in this paper began with identifying how the current notion of the interruption context inadequately deals with aspects pertaining to the relationship between the interrupter and interruptee including interaction histories and interruption content (what we referred to as the relational context).We then presented a theoretical framework that shows how individuals aim at reducing uncertainty of relational context to predict the value of an interruption. Utilizing this framework we suggest a research program in interpersonal interruption management that focuses on interrupteeside decision making and response behavior rather than allowing systems to make decisions and take action automatically on behalf of the user. Three key research areas emerge: 1) What is the interrelationship between cognitive, social, and relational context in interruption management decision making; 2) How is uncertainty of the relational context related to predicted interruption value (PIV) and 3) How can we design communication tools that optimize the presentation of relational context information to the interruptee so as to better support interruption handling decisions. We now present a research agenda organized around these three key areas. The research questions that arise in these areas are discussed below.

Inter-relationship between cognitive, social and relational contexts in interruption management decision making: In regards to assessing responsiveness to interruptions, the framework enables inquiry into how relational context impacts interruption management decision making. Little work has been undertaken to understand how cognitive, social, and relational context information collectively influence interruptibility. For example, we do not know how often people utilize relational context in their interruptibility decisions and how its importance compares with cognitive and social context. The need for improved understanding of how relational context is used in interruption management is of particular relevance to mobile communication technologies because 'communication tools' are also 'social relationship tools', currently the only information available to the callee pertaining to relational context is caller ID, and additional information about the caller can be provided relatively easily. Beyond the fact that people use practices such as turning off phones, ignoring calls and using caller ID to decide on response strategy, we have very little understanding about what motivates people's call handling decisions. In a recent field study, people's reasons for call handling decisions on cell phones were captured in situ using experience sampling methodology (ESM). The study provided empirical evidence that the relational context (who the caller is) plays an enormous role (87.4\%) in people's call handling decisions $(\mathrm{N}=834)$. It also highlighted how individuals routinely engage in uncertainty reduction (35.4\% of answered calls) to know what the call was about [16]. While this study points to the importance of relational context, richer understanding is required of relational context information that is limited to caller identification alone. Specifically, the following research questions need to be explored: 1) How and when do people use relational, cognitive, and social context in interruption management decision making? 2) What is the relationship between these three contexts? 3) Does relational context trump social and cognitive contexts in interruption management decision-making, if so when? 4) How important relational context is in making interruption management decisions? Exploring these research questions require a combination of both qualitative and quantitative methods. 
Uncertainty of relational context and its relationship to predicted interruption value (PIV): The theoretical framework developed proposes that reducing uncertainty around relational context leads to more accurate prediction of the interruption value. It is important to understand how uncertainty around relational context operates and how interruption value is predicted in current interruption management practices. This raises the following research questions in everyday communication 1) what information do interruptees use and/or desire to reduce uncertainty of an interruption? 2) What information do they use and/or desire to predict the value of an interruption? 3) How and when is information related to relational context perceived to be more beneficial to predicting the value of an interruption? 4) How does the need for this information vary with other social and cognitive contexts? 5) When do users want this information - do they want it ahead of a future interruption for planning purposes or just in time to make satisfying interruption management decision? 6) What level of granularity of this information is desired? How does this vary across different communication/collaborative tools? In addressing these questions we could employ both qualitative and quantitative methods as mentioned above. Data on user behavior can be obtained in several ways: interviews, surveys, and ESM [21] where user responses are captured in situ. It is paramount to ensure that the data provides insight on why users take certain actions to understand the impact of relational context in interruption decision making. Survey or interview methods can be used to explicitly ask the users questions to understand the rationale behind their behavior. Experience sampling methods where questions are asked in the context of the user behavior (such as handling an incoming phone call) helps in increased accuracy of recall.

Designing Interruption Management tools: A major challenge here is to meaningfully analyze and interpret findings from the above research areas in a way that informs the design of the interruption management support tool. While capturing relational context information that individual's are uncertain about is fairly straight forward, designers need to understand the tradeoffs in information presentation to minimize uncertainty and aid in interruption value prediction. While user studies may reveal many merits/demerits of information that can be used for uncertainty reduction and outcome-value estimation, designers should identify how much information should be presented without causing information overload. Further, we need to address the challenges in organizing and displaying information to support easy, quick and effective interruption management decision making. This presents the following design questions and challenges: 1) what are the tradeoffs in information presentation to the user to minimize uncertainty and maximize outcome value prediction? 2) How much information should be presented to minimize uncertainty and maximize outcome value prediction without causing information overload? and 3) How should information be organized and displayed to support easy, quick and effective interruption decision making?

The theoretical framework and research agenda presented here suggest new areas of enquiry and more importantly new approaches to the design of effective interruption management tools that aim to empower the interruptee in the emerging technology environment of next generation of interpersonal communication tools. Exploring interruption management from an interpersonal relational context perspective holds the promise of bringing valuable insight that has been lost in the previous research on human interruptibility.

\section{Acknowledgments}

This research is partially supported by the National Science Foundation (NSF CNS 0454081 and IIS 0534520).

\section{References}

[1] Avrahami, D., D. Gergle, S.E. Hudson and S. Kiesler ( 2007) Improving the Match between Callers and Receivers: A Study on the Effect of Contextual Information on Cell Phone Interruptions. Behaviour and Information Technology.

[2] Avrahami D. \& Hudson S. E. (2006) Responsiveness in instant messaging: predictive models supporting interpersonal communication, in: Human Factors in Computing System: Proceedings of $C H I^{\prime} 06$, New York: ACM Press 731740

[3] Bailey B. P., Konstan J. A. \& Carlis J. V. (2001) The effects of interruptions on task performance, annoyance, and anxiety in the user interface, in: M.Hirose (Ed.) HumanComputer Interaction - INTERACT 2001 Conference Proceedings Amsterdam:IOS Press,593-601

[4] Barrett, L.F., Barrett, D.J., "An Introduction to Computerized Experience Sampling in Psychology," Social Science Computer Review, Vol. 19, No. 2, (Summer 2001)

[5] Berger, C. R., \& Calabrese, R. J. (1975). Some exploration in initial interaction and beyond: Toward a developmental theory of communication. Human Communication Research, 1, 99-112.

[6] Broadbent, D. E.(1958).Perception and Communication. London: Pergamon Press

[7] Burke, K. (1966). Language as symbolic action: Essays on life, literature, and method. Berkeley: University of California Press.

[8] Czerwinski M., Cutrell E. \& Horvitz E. (2000) Instant messaging: Effects of relevance and time, in: S.Turner \& 
P.Turner (Eds.),People \&ComputersXIV:Proceedings of HCI 2000, Vol. 2, British Computer Society, 71-76

[9] Dabbish L. A ( 2006) Coordinating Initiation and Response in Computer-Mediated Communication, $\mathrm{PhD}$ Dissertation, Carnegie Mellon University

[10] Dey, A. K. (2001) Understanding and using context, Personal and Ubiquitous Computing, 5(1):4-7.-

[11] Erickson, T. (2001) Ask not for whom the cell phone tolls: Some problems with the notion of context-aware computing. Communications of the ACM.

[12] Fogarty J., Hudson S. E., Atkeson C. G., Avrahami D., Forlizzi J., Kiesler S., Lee J. C. \& Yang J.(2005) Predicting human interruptibility with sensors, ACM Transactions on Computer-Human Interaction, 12(1), 119-146

[13] Gillie, T., \& Broadbent, D. E. (1989). What makes interruptions disruptive? A study of length, similarity, and complexity. Psychological Research, 50, 243-250.

[14] Goffman, E. (1959) The Presentation of Self in Everyday Life, London: Penguin.

[15] González V. M. \& Mark G. (2004) "Constant, constant, multi-tasking craziness": Managing multiple working spheres, in: Human Factors in Computing Systems: Proceedings of CHI'04, New York: ACM Press, 113-120

[16] Grandhi, S.,A., Laws N., Amento, B., and Jones, Q., "The importance of who" and "what" in Interruption Management: Empirical Evidence from a Cell Phone Use Study", AMCIS 2008 Proceedings.

[17] Gruen D. M. (1996) The Role of External Resources in the Management of Multiple Activities, PhD Dissertation, University of California at San Diego

[18] Ho J. \& Intille S. S. (2005) Using context-aware computing to reduce the perceived burden of interruptions from mobile devices, in: Human Factors in Computing Systems: Proceedings of CHI'05, New York: ACM Press, 909-918

[19] Horvitz E., Koch P. \& Apacible J. (2004) BusyBody: Creating and fielding personalized models of the cost of interruption, in: Proceedings of the ACM Conference on Computer Supported Cooperative Work (CSCW'04), New York: ACM Press, 507-510

[20] Horvitz E., Koch P., Sarin R., Apacible J. \& Subramani M. (2005) Bayesphone: Precomputation of context-sensitive policies for inquiry and action in mobile devices, in: L. Ardissono, P. Brna, A. Mitrovic (Eds.), User Modeling 2005: Proceedings of 10th International Conference(UM 2005), Berlin: Springer-Verlag,251-260

[21] Hudson J. M., Christensen J., Kellogg W. A. and Erickson T. (2002) "I'd be overwhelmed, but it's just one more thing to do": Availability and interruption in research management. In: Human Factors in Computing Systems: Proceedings of CHI'02. ACM Press, 97-104

[22] Iqbal S. T. \& Bailey B. P. (2005) Investigating the effectiveness of mental workload as a predictor of opportune moments for interruption, in: Human Factors in Computing Systems: Proceedings of CHI'05: Extended Abstracts. New York: ACM Press, 1489-1492
[23] Kahneman, D. (1973). Attention and effort. Englewood Cliffs, NJ: Prentice-Hall.

[24] Kern N., Antifakos S., Schiele B. \& Schwaninger A. (2004) A model for human interruptability: Experimental evaluation and automatic estimation from wearable sensors, in: Proceedings of the 8th International Symposium on Wearable Computers, Los Alamitos: IEEE Computer Society, 158-65

[25] Maglio P. P. \& Campbell C. S. (2000) Tradeoffs in displaying peripheral information, in: Proceedings of the $\mathrm{CHI}$ 2000 conference on Human factors in computing systems, New York: ACM Press, 241-248

[26] Marti S., Schmandt C., (2005), Physical Embodiments for Mobile Communication Agents.In Proceedings of UIST 2005, Seattle, Washington, October 23-26

[27] McFarlane D. C. \& Latorella K. A. (2002) The scope and importance of human interruption in human-computer interaction design, Human-Computer Interaction, 17 (1),

[28] Milewski A. (2006) Interruption Management and Telephone Call Screening, International Journal of Human Computer Interaction, Vol. 20, No. 1, pp 19-33

[29] O'Conaill B. \& Frohlich D. (1995) Timespace in the workplace: Dealing with interruptions, in: Human Factors in Computing Systems: CHI'95 Companion, New York: ACM Press, 262-263

[30] Pedersen, E.R. (2001). Calls.calm: Enabling Caller and Callee to Collaborate. Extended Proceedings of CHI '01, Seattle, pp 235-236

[31] Perlow, A., L., "The time famine: Toward a sociology of work time" Administrative Science Quarterly; Mar 1999; 44, 1; ABI/INFORM Global pg. 57

[32] Schilit, B. and Theimer, M., Disseminating active map information to mobile hosts. IEEE Network, 8(5):22-32, 1994.

[33] Schilit, B., Adams, N., and Want, R. Context-aware computing applications. In Proceedings of the $1 \mathrm{st}$ International Workshop on Mobile Computing Systems and Applications, Los Alamitos, CA, 1994. IEEE.

[34] Speier C., Valacich J. S. \& Vessey I. (1997) The effects of task interruption and information presentation on individual decision making, in: K. Kumar \& J. I. DeGross (Eds.), Proceedings of the XVIII International Conference on Information Systems, Atlanta: Association for Information Systems, 21-36

[35] Sunnafrank, M. (1986). Predicted outcome value during initial interactions: A reformulation of Uncertainty Reduction Theory. Human Communication Research, 13(1), 3-33.

[36] Szóstek A. M. \& Markopoulos P. (2006) Factors defining face-to-face interruptions in the office environment, in: CHI '06 Extended Abstracts on Human Factors in Computing Systems, New York: ACM Press, 1379-1384

[37] Zijlstra, F. R. H., \& Roe, R. A. (1999). Temporal factors in mental work: Effects of interrupted activities. Journal of Occupational and Organizational Psychology 72, 163-185 